\title{
Molecular aspects of the potato - Phytophthora infestans interaction
}

\section{CORNÉ M.J. PIETERSE, PIERRE J.G.M. DE WIT and FRANCINE P.M. GOVERS}

Department of Phytopathology, Agricultural University, Binnenhaven 9, P.O. Box 8025, 6700 EE Wageningen, the Netherlands

Accepted 5 november 1991

\begin{abstract}
The fungal pathogen Phytophthora infestans is the causal organism of late blight, one of the most devastating diseases of potato. In the past, various aspects of the potato- $P$. infestans interaction have been studied extensively. In this paper we briefly review the current knowledge of the molecular events associated with the interaction and in addition we discuss a new approach for analyzing the molecular basis of pathogenicity of $P$. infestans.
\end{abstract}

Additional keywords: oomycetes, pathogenicity, potato late blight.

\section{Introduction}

Potato late blight caused by the fungus Phytophthora infestans (Mont.) de Bary (Oomycetes) is one of the most important diseases of potato (Solanum tuberosum (L.)). Leaves and tubers of susceptible cultivars become readily infected by this pathogen. The fungus spreads rapidly through the plant tissue, causing a destructive necrosis.

The disease cycle starts when a sporangium comes into contact with a potato leaf or tuber. On moist surfaces, the sporangium germinates directly or develops a zoosporangium with motile biflagellate zoospores which germinate after encystment. Germinating sporangia and cysts form appressoria, and infection tubes emerging from the appressoria penetrate the host cells (Pristou and Gallegly, 1954). In leaves, penetration occurs preferably in the region of stomata, immediately adjacent to stomatal guard cells (Gees and Hohl, 1988). The infection tubes enter the epidermal cell and form hyphal structures. The hyphae grow into the mesophyll cellolayer, both intra- and intercellularly, occasionally forming haustoria (Coffey and Wilson, 1983). After colonization of the leaf tissue, sporangiophores emerge from the stomata, forming thousands of sporangia which can be dispersed by wind or rain and eventually reinfect plants nearby or over long distances. In leaves, the first macroscopic sign of lesion development is the appearance of a water-soaked area. While the fungus grows and sporulates at the advancing edges of the developing lesion, the center of the spreading lesion becomes necrotic. This can eventually lead to complete destruction of foliage and consequent reduction in tuber yields. Tuber infection itself can also cause severe losses, especially during storage.

$P$. infestans is a hemibiotrophic pathogen with a rather narrow host range, all host plants being Solanaceae. From the crop plants, potato is economically the most important host but also tomato (Lycopersicon esculentum (Mill.)) can be infected. In addition, $P$. infestans is capable of attacking wild relatives of potato and tomato. The specificity of 
$P$. infestans for Solanaceae, in particular potato, implies that there is a specific recognition mechanism between pathogen and host which consequently involves the exchange of signals between the two organisms. On the one hand, recognition of $P$. infestans by the host leads to the induction of defence responses directed towards the pathogen. On the other hand, it is very likely that contact of the pathogen with a potential host induces responses in the pathogen which are required for pathogenesis. Thus in both the host plant and the pathogen, the pathogenic process is likely to induce the expression of many genes.

The extremely destructive nature of the potato late blight disease and its world-wide distribution make the potato- $P$. infestans interaction a topic of research for many plant pathologists. A considerable amount of research has been dedicated to date on various aspects of the potato- $P$. infestans relationship (Ingram and Williams, 1991). Here we review the current state of the art concerning the genetic and molecular aspects of this plant-pathogen interaction.

\section{The genetic basis of resistance to Phytophthora infestans}

Genetically controlled disease resistance to plant pathogens can be classified as either vertical or horizontal resistance. By classical breeding both types of resistance to $P$. infestans have been introduced into potato. In the potato- $P$. infestans pathosystem, vertical resistance, also called race-specific resistance, is based on a gene-for-gene relationship in which presumably the products of avirulence genes of the pathogen, the so-called race-specific elicitors, interact directly or indirectly with the products of the matching major resistance genes (R-genes) of the plant. This results in the triggering of a hypersensitive response (HR). Invaded host cells and cells surrounding the infected cells die rapidly. The biotrophic fungus can not grow further and this finally results in an incompatible interaction. Races lacking avirulence genes corresponding to R-genes in the host do not trigger HR. They are able to colonize the plant successfully, resulting in a compatible interaction.

Little is known about the genetic and molecular basis of avirulence/virulence in $P$. infestans and race-specific resistance/susceptibility in potato. In most plant pathogens avirulence is dominant over virulence but there are exceptions (Shaw, 1991). Spielman et al. (1989), who analyzed the segregation of virulence loci in the progeny of genetic crosses, showed that in $P$. infestans avirulence towards the potato resistance genes R3 and the tomato resistance gene $\mathrm{Ph} 1$ is dominant. On the other hand they found that for R2 and R4 avirulence inherits as a recessive trait. Whether dominant inhibitor genes, which mask the expression of avirulence, are involved is not known. Vertical resistance, governed by the so-called R-genes, is single gene based. To date, 11 dominant R-genes, all originating from Solanum demissum (L.) have been introduced into the cultivated potato by breeding (Wastie, 1991). Mapping of R-genes in potato and virulence/avirulence genes in $P$. infestans is in progress in several laboratories. Gebhardt and coworkers have recently determined the location of resistance gene R1 on a genetic map of potato (Leonards-Schippers et al., 1992). This is a first step towards the cloning of R-genes. Another way to clone Rgenes is by means of transposon tagging, an approach pursued by Peirera et al. (this volume). The isolation and characterization of R-genes and the corresponding avirulence genes from $P$. infestans will lead to a better understanding of the mechanisms involved in the induction of the defence responses.

In contrast to vertical resistance in which a single $\mathrm{R}$-gene is sufficient to protect the plant completely against particular races of $P$. infestans, horizontal resistance, also called race- 
nonspecific resistance or field resistance, is assumed to be multiple gene based. In horizontal resistant cultivars the infection efficiency might be lower, the tissue colonization slower and the sporulation rate reduced (Umaerus, 1970). The level of field resistance varies among cultivars. This type of resistance is durable and thus commercially more attractive than race-specific resistance. In the latter, new virulent races evolve rapidly, rendering the $\mathrm{R}$ genes ineffective. Breeding efforts therefore aim at introducing durable field resistance into commercial potato cultivars. The molecular and genetic basis underlying field resistance is basically unknown. Therefore, breeders have to rely on empirical methods.

\section{The plant response}

The development of the potato late blight disease is dependent on the aggressiveness of the pathogen and the defence response of the host. Spores of all races germinate with equal efficiency on resistant and susceptible cultivars and also the frequency of penetration is comparable (Gees and Hohl, 1988). In compatible as well as incompatible interactions, the initially invaded host cells react hypersensitively and produce callose-like material within a few hours after inoculation (Cuypers and Hahlbrock, 1988). However, in the incompatible interaction, the epidermal and mesophyll cells adjacent to the invaded cells become necrotic within 5 hours after inoculation, whereas in the compatible interaction these cells do not become necrotic within the first 12-24 hours (Cuypers and Hahlbrock, 1988). Thus in general it can be stated that in comparison with compatible interactions, in incompatible interactions the host cells respond to $P$. infestans more rapidly by triggering HR. Upon pathogen attack in resistant as well as susceptible plants numerous genes are activated, leading to the accumulation of pathogenesis-related (PR) proteins and secondary metabolites such as phytoalexins and lignin.

Phytoalexins are antimicrobial molecules which are produced by the plant upon infection, elicitor treatment or wounding. In potato tubers, phytoalexin production occurs primarily via the terpenoid biosynthesis pathway, resulting in sesquiterpenoids such as rishitin, phytuberin and lubimin (Kuc, 1982). Upon infection of potato tubers with $P$. infestans, sesquiterpenoid phytoalexins are produced in healthy tissue around the infection site and are then transported into browning cells where they can accumulate to high concentrations (Sato et al., 1971). Rohwer et al. (1987) studied the phytoalexin accumulation in a compatible and an incompatible interaction. In potato tubers, they found a more rapid accumulation of sesquiterpenoid phytoalexins during the first 24-48 hours of infection in the incompatible interaction compared to compatible one. In infected leaves no sesquiterpenoid phytoalexins are detectable. This suggests that at least in leaves, sesquiterpenoid phytoalexins are not necessary for defence against $P$. infestans. One of the key enzymes in the sesquiterpenoid phytoalexin biosynthesis pathway is 3-hydroxy-3-methylglutaryl coenzyme A reductase (HMGR) which catalyzes the conversion of 3-hydroxy-3-methylglutaryl coenzyme A to mevalonic acid, the rate-limiting step in terpenoid biosynthesis. HMGR activity increases in response to wounding, infection with $P$. infestans and treatment with arachidonic acid, an elicitor of the synthesis of sesquiterpenoid phytoalexins. Recently, Yang et al. (1991) showed that increased activity of HMGR in potato tubers upon infection with Erwinia carotovora, wounding or elicitor treatment is due to induced gene expression. In potato HMGR is encoded by a small gene family of which one isogene is activated upon pathogen attack and another upon wounding. This indicates that the 
signals leading to activation of HMGR genes upon pathogen challenge and wounding are different.

Another major plant response to pathogen attack is the induction of expression of the phenylalanine ammonia-lyase (PAL) and 4-coumarate:CoA ligase (4-CL) genes. PAL and 4-CL are both key enzymes in the phenylpropanoid pathway, the biosynthesis pathway of lignin and other wall-bound phenolics. Fritzemeier et al. (1987), who studied the PAL and 4-CL gene expression in potato, found that in incompatible interactions the expression of both genes is rapidly induced within several hours post-inoculation, whereas in compatible interactions this is slightly delayed. This difference in timing of PAL mRNA accumulation was also demonstrated by Cuypers et al. (1988), who used the in situ hybridization technique. In an incompatible interaction, a clear halo of accumulating PAL mRNA around the penetration site appeared within 3 hours after inoculation and disappeared within the following 3 hours. In the compatible interaction, however, the halo of accumulating PAL mRNA did not appear before 6 hours post-inoculation. In potato, lignin and other soluble and wall-bound phenolics are the defence related endproducts of the phenylpropanoid pathway. Ampomah and Friend (1988) found a correlation between PAL activity, deposition of lignin or lignin-like material and susceptibility to $P$. infestans infection in potato tuber discs. In tuber discs of cultivars with a high degree of field resistance more lignin and lignin-like material was deposited than in tuber discs of susceptible cultivars. Treatment with an inhibitor of PAL decreased the deposition of lignin and lignin-like material and the tuber discs became more susceptible to fungal infection. Thus the endproducts of the phenylpropanoid pathway may play a role in plant defence but their contribution to the ultimate resistance reaction remains to be resolved.

PR proteins are a characteristic group of plant proteins which accumulate after infection, elicitor treatment and sometimes during senescence. Kombrink et al. (1988) identified a number of PR proteins which accumulate in the intercellular space of potato leaves following inoculation with $P$. infestans. Eight of these proteins have been purified. Six appeared to have chitinase activity and two 1,3- $\beta$-glucanase activity. Taylor et al. (1990) isolated a cDNA clone of a potato gene which shows induced expression very early after infection by $P$. infestans and which has a striking homology with a gene encoding a heat shock protein in soybean. The expression pattern of this gene, which was designated PR1, is similar to that of the PAL and 4-CL genes in both compatible and incompatible interactions. Matton and Brisson (1989) selected two other cDNA clones of potato genes encoding PR proteins. The expression of these genes reached a maximum within 24 hours after elicitor treatment. The two proteins encoded by these genes differ by only six amino acids and show a high degree of similarity with PR protein sequences from pea and parsley. The induced expression of genes encoding PR proteins and the subsequent accumulation of these proteins upon infection suggest that they are part of a plant defence mechanism. Some PR proteins, e.g., 1,3- $\beta$-glucanases and chitinases can potentially degrade cell walls of fungi. A major component of the $P$. infestans cell wall is $1,3-\beta$-glucan, but chitin is lacking. Therefore only $1,3-\beta$-glucanases are potentially capable of hydrolyzing the cell wall of $P$. infestans. However, there is no direct evidence that these proteins, as well as the other identified PR proteins, are involved in resistance against $P$. infestans. The only protein that has been described to have inhibitory activity towards $P$. infestans is the thaumatin-like protein osmotin which was isolated from tobacco and tomato (Woloshuk et al., 1991). Purified osmotin causes lysis of sponrangia and inhibition of hyphal growth in vitro, which indicates that osmotin might have a role in the plant defence mechanism. 
In both compatible and incompatible interactions similar plant responses are observed but their timing is different. Whether this difference in timing determines the ultimate outcome of the resistance reaction is not known. Also the relative contribution of the various synthesized compounds to the resistance reaction is unknown. It is likely that a combination of compounds, produced at the right time and the right place, ultimately determines the outcome of the resistance reaction. However, it can not be ruled out that other, so far unknown, defence mechanisms are involved.

\section{Pathogenicity of Phytophthora infestans and characterization of putative pathogeni- city genes}

Pathogenicity is a complex process that most likely requires the expression of particular pathogenicity genes, i.e., genes of the pathogen which are essential for the establishment and maintenance of a compatible interaction. Pathogenicity factors such as cutinases, phytotoxins and cell wall degrading enzymes are important for pathogenicity of various plant pathogenic fungi. For $P$. infestans, however, it is unlikely that these pathogenicity factors have an important function in the interaction. $P$. infestans forms an appressorium and penetrates the host cells with physical forces. Enzymatic breakdown of the cuticle and epidermal cell walls is probably not required. Since $P$. infestans is a hemibiotrophic pathogen it is unlikely that it produces toxins which kill the host cells immediately. This biotrophic nature also suggests that the cell wall degrading enzyme system of $P$. infestans is under tight control. The cell walls must be degraded to some extent so that the fungal hyphae can grow through the tissue, but the host cells should not be destroyed. Several hydrolytic enzymes have been isolated from culture filtrate of $P$. infestans. Among those are endocellulases, B-glucosidases, 1,3-B-glucanases, polygalacturonases and galactanases (Jarvis et al., 1981; Bodenmann et al., 1985), but none of the hydrolytic enzymes show massive cell wall degrading activity.

To date, hardly anything is known about the molecular basis underlying pathogenicity of $P$. infestans. It can be assumed that, like in other plant-microbe interactions (e.g., dicots-Agrobacterium, legumes-Rhizobium spp. and pea-Fusarium f.sp. pisi), signal molecules originating from the host induce the expression of genes in the pathogen which are necessary for pathogenesis. Thus one way to gain more insight in the pathogenicity process is by characterizing $P$. infestans genes which show induced expression during a compatible interaction with the host plant. Once these genes are isolated, their role in pathogenicity can be studied in more detail. In order to isolate these putative pathogenicity genes we screened a genomic library of $P$. infestans by differential hybridization. ${ }^{32} \mathrm{P}$ labeled cDNA derived from $m$ RNA isolated from the potato- $P$. infestans interaction and ${ }^{32} \mathrm{P}$-labeled cDNA synthesized on mRNA of the fungus grown in vitro were used as probes. Clones that hybridized specifically to the interaction cDNA probe were selected and genes located on these clones were further characterized. In order to confirm that the expression of the selected genes is induced in planta, expression analyses were performed. Northern blots containing total RNA from non-infected leaves, from infected leaves of different stages after inoculation and from $P$. infestans grown in vitro, were hybridized with radioactively labeled probes of three putative in planta induced genes (Fig. 1). To determine the proportion of fungal RNA in the RNA preparation isolated from infected leaves, the northern blots were hybridized with a probe of a constitutively expressed $P$. infestans gene encoding actin (act A, described and provided by Unkles et al., 1991). Comparison of 


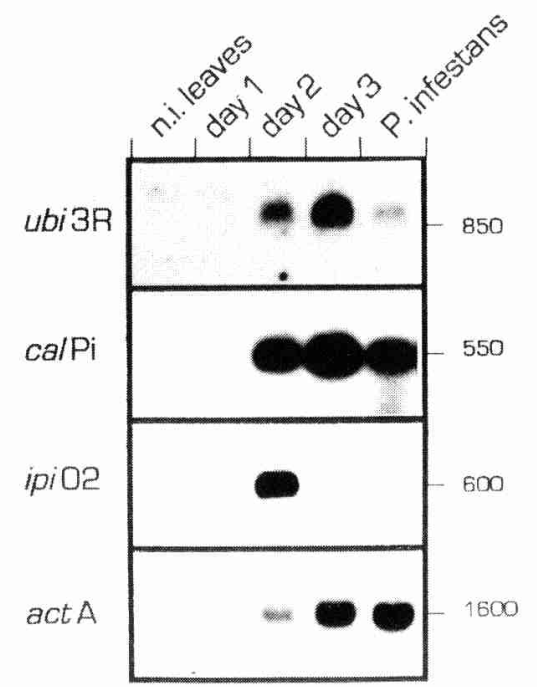

Fig. 1. Autoradiographs of Northern blots containing $15 \mu \mathrm{g}$ of total RNA isolated from non-infected potato leaves (n.i. leaves), from colonized potato leaves 1-3 days post-inoculation (day 1-3) and from Phytophthora infestans grown in vitro ( $P$. infestans). The northern blots were hybridized with a probe made from the gene-specific promoter region of ubi3R and from the coding regions of cal $\mathrm{Pi}$, ipiO2 and act $\mathrm{A}$.

the hybridization signal in the lanes containing RNA from the fungus grown in vitro and RNA from infected leaves indicates that the expression of the three genes is higher when the fungus is growing in the host. One of the three genes, designated ipiO2, shows a transient expression pattern. This gene is highly expressed up to two days after inoculation whereas one day later the expression is much lower. In RNA preparations from the fungus grown in vitro, the ipiO2 transcript is not detectable at all. From the other two genes, designated ubi3R and calPi respectively, transcripts are detectable in the in vitro grown fungus but in planta the expression is several-fold higher.

From DNA sequence analyses it appeared that $u b i 3 \mathrm{R}$ codes for polyubiquitin (Pieterse et al., 1991) and calPi for calmodulin. Ubi3R and calPi are highly homologous with respectively polyubiquitin and calmodulin genes of other eukaryotic organisms, e.g., human (95\% resp. $92 \%$ ) and potato (95\% resp. $87 \%$ ). Ubiquitin plays a key role in several cellular processes such as selective degradation of intra-cellular proteins, maintenance of chromatin structure, regulation of gene expression and modification of cell surface receptors (Monia et al., 1990). Calmodulin is a calcium binding protein which is known to play an essential role in basic cellular processes such as signal transduction, ion transport and cytoskeleton function. After binding of calcium to calmodulin, the protein undergoes a conformational change which enables it to interact with calmodulin dependent enzyme molecules. Many enzymes are known to be regulated by the $\mathrm{Ca}^{2+}-$ calmodulin complex (Cheung, 1980). Because of these basic functions it is not surprising that ubi3R and calPi are expressed in the in vitro grown fungus as well. Induced expression of these genes during growth in planta suggests that higher levels of these proteins are required for pathogenicity, but the precise role in the pathogenicity process remains to be solved. 
The ipi $\mathrm{O} 2$ gene codes for a protein of 152 amino acids. The nucleotide sequence nor the amino acid sequence of ipiO2 has homology with sequences present in any nucleotide- or protein database. A putative signal peptide containing a hydrophobic core is present at the $\mathrm{N}$-terminal part of the protein indicating that the protein is excreted. Furthermore the protein has a putative 'cell attachment' sequence (recognizable by an 'RGD' tripeptide), which has been shown to play a role in cell adhesion of several mammalian proteins, and a potential $\mathrm{N}$-glycosylation site. The transient expression pattern of the ipiO2 gene in infected leaves suggests that the protein has a function in specific steps of the infection process. We will concentrate on studying the function of the ipiO2 protein in pathogenesis. Feasible approaches are localization of the ipiO2 transcript, gene disruption and inhibition of translation of ipiO2 mRNA via anti-sense RNA.

\section{Concluding remarks}

Although there have been extensive studies on various aspects of the interaction between $P$. infestans and potato there are still considerable gaps in our knowledge. For example, the processes involved in molecular signalling which leads to compatibility and incompatibility are poorly understood. Potato resistance genes, which are certainly involved in this signalling, have to be isolated and characterized in order to get a better understanding of the gene-for-gene relationship.

Of the host plant potato, all the defence responses which have been studied at the molecular level can more or less be mimicked with non-race-specific elicitors and apparently there is no association with R-gene mediated resistance. The significance of these responses in plant defence is not very well understood yet and further investigations are needed.

Of the pathogen $P$. infestans, the molecular basis of pathogenicity has hardly been studied. In the past the lack of a transformation system made it difficult to address questions in this direction. Recently Judelson et al. (1991) successfully transformed P. infestans. Although the transformation efficiency is still very low, this achievement will surely stimulate efforts to isolate and characterize pathogenicity and avirulence genes of the pathogen.

\section{References}

Ampomah, Y.A. \& Friend, J., 1988. Insoluble phenolic compounds and resistance of potato tuber disc to Phytophthora and Phoma. Phytochemistry 27: 2533-2541.

Bodenmann, J., Heiniger, U. \& Hohl, H.R., 1985. Extracellular enzymes of Phytophthora infestans: endo-cellulase, B-glucosidases, and 1,3-B-glucanases. Canadian Journal of Microbiology 31: 75-82.

Cheung, W.Y., 1980. Calmodulin - an introduction. In: Cheung, W. Y. (Ed.), Calcium and cell function, Vol. I, Calmodulin. Academic Press, New York. p. 2-12.

Coffey, M.D. \& Wilson, U.E., 1983. Histology and cytology of infection and disease caused by Phytophthora. In: Erwin, D.C., Bartnicki-Garcia, S. \& Tsao, P.H. (Eds), Phytophthora: Its biology, taxonomy, ecology, and pathology. American Phytopathological Society, St. Paul, Minnesota. p. 289-301.

Cuypers, B. \& Hahlbrock, K., 1988. Immunohistochemical studies of compatible and incompatible interactions of potato leaves with Phytophthora infestans and of the non-host response to Phytophthora megasperma. Canadian Journal of Botany 66: 700-705.

Cuypers, B., Schmelzer, E. \& Hahlbrock, K., 1988. In situ localization of rapidly accumulated phenylalanine ammonia lyase mRNA around penetration sites of Phytophthora infestans in potato leaves. Molecular Plant-Microbe Interactions 1: 157-160.

Fritzemeier, K-H., Cretin, C., Kombrink, E., Rohwer, F., Taylor, J., Scheel, D. \& Hahlbrock, K., 1987. Transient induction of phenylalanine ammonia-lyase and 4-coumarate:CoA ligase mRNAs 
in potato leaves infected with virulent or avirulent races of Phytophthora infestans. Plant Physiology 85: 3441 .

Gees, R. \& Hohl, H.R., 1988. Cytological comparison of specific (R3) and general resistance to late blight in potato leaf tissue. Phytopathology 78: 350-357.

Ingram, D.S. \& Williams, P.H. (Eds), 1991. Advances in plant pathology, Vol. 7, Phytophthora infestans, the cause of late blight of potato. Academic Press, London, San Diego.

Jarvis, M.C., Threlfall, D.R. \& Friend, J., 1981. Potato cell wall polysaccharides: degradation with enzymes from Phytophthora infestans. Journal of Experimental Botany 32: 1309-1319.

Judelson, H.S., Tyler, B.M. \& Michelmore, R.W., 1991. Transformation of the oomycete pathogen, Phytophthora infestans. Molecular Plant-Microbe Interactions 4: 602-607.

Kombrink, E., Schröder, M. \& Hahlbrock, K., 1988. Several 'pathogenesis-related' proteins in potato are 1,3- $\beta$-glucanases and chitinases. Proceedings of the National Academy of Science USA 85: $782-786$.

Kuc, J., 1982. Phytoalexins from the Solanaceae. In: Bailey, J.A. \& Mansfield, J.W. (Eds), Phytoalexins. Blackie, Glasgow, London. p. 81-105.

Leonards-Schippers, C., Gieffers, W., Salamini, F. \& Gebhardt, C., 1992. The R1 gene conferring race-specific resistance to Phytophthora infestans in potato is located on potato chromosome V. Molecular and General Genetics 233: 278-283.

Matton, D.P. \& Brisson, N., 1989. Cloning, expression, and sequence conservation of pathogenesisrelated gene transcripts of potato. Molecular Plant-Microbe Interactions 2: 325-331.

Monia B.P., Ecker, D.J. \& Crooke, S.T., 1990. New perspectives on the structure and function of ubiquitin. Bio/technology 8: 209-215.

Pieterse, C.M.J., Risseeuw, E.P. \& Davidse, L.C., 1991. An in planta induced gene of Phytophthora infestans codes for ubiquitin. Plant Molecular Biology 17: 799-811.

Pristou, R. \& Gallegly, M.E., 1954. Leaf penetration by Phytophthora infestans. Phytopathology 44: $81-86$.

Rohwer, F., Fritzemeier, K-H., Scheel, D. \& Hahlbrock, K., 1987. Biochemical reactions of different tissues of potato (Solanum tuberosum) to zoöspores or elicitors from Phytophthora infestans. Planta 170: 556-561.

Sato, N., Kitazawa, K. \& Tomiyama, K., 1971. The role of rishitin in localizing the invading hyphae of Phytophthora infestans in infection sites at the cut surfaces of potato tubers. Physiological Plant Pathology 1: 289-295.

Shaw, D.S., 1991. Genetics. In: Ingram, D.S \& Williams, P.H. (Eds), Advances in plant pathology, Vol. 7, Phytophthora infestans, the cause of late blight of potato. Academic Press, London, San Diego. p. 131-170.

Spielman, L.J., McMaster, B.J. \& Fry, W.E., 1989. Dominance and recessiveness at loci for virulence against potato and tomato in Phytophthora infestans. Theoretical and Applied Genetics 77: $832-838$.

Taylor, J.L., Fritzemeier, K.-H., Häuser, I., Kombrink, E., Rohwer, F., Schröder, M., Strittmatter, G. \& Hahlbrock, K., 1990. Structural analysis and activation by fungal infection of a gene encoding a pathogenesis-related protein in potato. Molecular Plant-Microbe Interactions 3: 72-77.

Umaerus, V., 1970. Studies on field resistance to Phytophthora infestans. 5. Mechanisms of resistance and application to potato breeding. Zeitschrift für Pflanzenzüchtung 63: 1-23.

Unkles, S.E., Moon, R.P., Hawkins, A.R., Duncan, J.M. \& Kinghorn, J.R., 1991. Actin in the oomycetous fungus Phytophthora infestans is the product of several genes. Gene 100: 105-112.

Wastie, R.L., 1991. Breeding for resistance. In: Ingram, D.S \& Williams, P.H. (Eds), Advances in plant pathology, Vol. 7, Phytophthora infestans, the cause of late blight of potato. Academic Press, London, San Diego. p. 193-224.

Woloshuk, C.P., Meulenhoff, E.J.S, Sela-Buurlage, M., Van den Elzen, P.J.M. \& Cornelissen, B.J.C., 1991. Pathogen-induced proteins with inhibitory activity toward Phytophthora infestans. The Plant Cell 3: 619-628.

Yang, Z., Park, H., Lacy, G.H. \& Cramer, C.L., 1991. Differential activation of potato 3-hydroxy-3methylglutaryl coenzyme A reductase genes by wounding and pathogen challenge. The Plant Cell 3: $397-405$. 